\title{
Le Fort I osteotomy as treatment for traumatic class III malocclusion caused by Le Fort III fracture: A case
} report

\author{
Han Byeol Jin, Jee Hyeok Chung, \\ Kyung Sik Kim, Seung Hong Kim, \\ Joon Choe, Jeong Yeol Yang
}

Department of Plastic and Reconstructive Surgery, Myongji Hospital, Goyang, Korea
Trauma in the modern society is characterized by multiple injuries, and the several comorbidities are often accompanied by facial bone fracture. The types of multiple facial bone fractures vary from Le Fort to panfacial fracture. Le Fort fracture, which can cause problems, such as facial disfigurement, functional impairment of mastication, malocclusion and speech abnormalities, is a challenging case for plastic surgeons. The purpose of treatment for patients with malocclusion due to Le Fort fracture is to maintain and restore both function and aesthetics. The author reports a case of Le Fort I osteotomy as a surgical correction of traumatic class III malocclusion due to Le Fort III fracture.

Keywords Le Fort osteotomy / Le Fort fracture / Malocclusion

\section{INTRODUCTION}

Recently, facial bone fracture is characterized by multiple injuries, and its surgery could be delayed due to the associated morbidity and mortality. In addition, even if open reduction is performed at an appropriate time, if the fracture fragments are comminuted, reduction of the fracture to its anatomical position before trauma is difficult, and firmly fixing it is difficult. Delayed surgery and inadequate reduction are prone to malunion of the fracture and other functional problems [1-3]. The types of multiple facial bone fractures vary from Le Fort fracture to panfacial fracture. Le Fort fracture, which can cause facial disfigurement, functional impairment of mastication, malocclusion, and speech abnormalities, is a challenging case for plastic surgeons. The healing process of this type of fracture is not normal due to the contraction force of the masti-

Received: Sep 3, 2018 Revised: Sep 20, 2018 Accepted: Sep 27, 2018 Correspondence: Jee Hyeok Chung Department of Plastic and Reconstructive Surgery, Myongji Hospital, 55 Hwasu-ro 14beon-gil, Deokyang-gu, Goyang 10475, Korea

Tel: +82-31-810-6830, Fax: +82-31-810-6837, E-mail: gabriel@mjh.or.kr

Copyright @ 2019 The Korean Society for Aesthetic Plastic Surgery.

This is an Open Access article distributed under the terms of the Creative Commons Attribution Non-Commercial License (http://creativecommons.org/licenses/by-nc/4.0/) which permits unrestricted non-commercial use, distribution, and reproduction in any medium, provided the original work is properly cited. $\quad$ www.e-aaps.org catory muscles in the jaw and oral system, which may result in malocclusion. Therefore, the purpose of the treatment of Le Fort fractures is to maintain and restore both function and aesthetics. The author reports a case of Le Fort I osteotomy as a surgical correction of traumatic class III malocclusion due to Le Fort III fracture.

\section{CASE REPORT}

A 54-year-old male patient complaining of disfigured facial appearance and discomfort due to malocclusion visited the Department of Plastic Surgery, Myongji Hospital. The patient had Le Fort II and III fractures due to a traffic accident 2 years prior and only underwent intermaxillary fixation at that time. His preoperative gross appearance showed midface depression, concave facial profile, and anteroposterior discrepancy of the upper and lower lips (Fig. 1). Preoperative cephalometric analysis showed skeletal class III malocclusion, and the Frankfort horizontal to $\mathrm{AB}$ angle was larger than normal. In addition, inadequate position of maxilla with depression, midline shifting, and canting were observed (Fig. 2). Preoperative intraoral evaluation showed class III malocclusion, crossbite, and midline shift to left of the maxillary plane (Fig. 3). Computed tomography scan revealed displacement of the maxilla due to inadequate reduction and fixation at the time of injury.

The author considered the patient's facial appearance, occlusion 

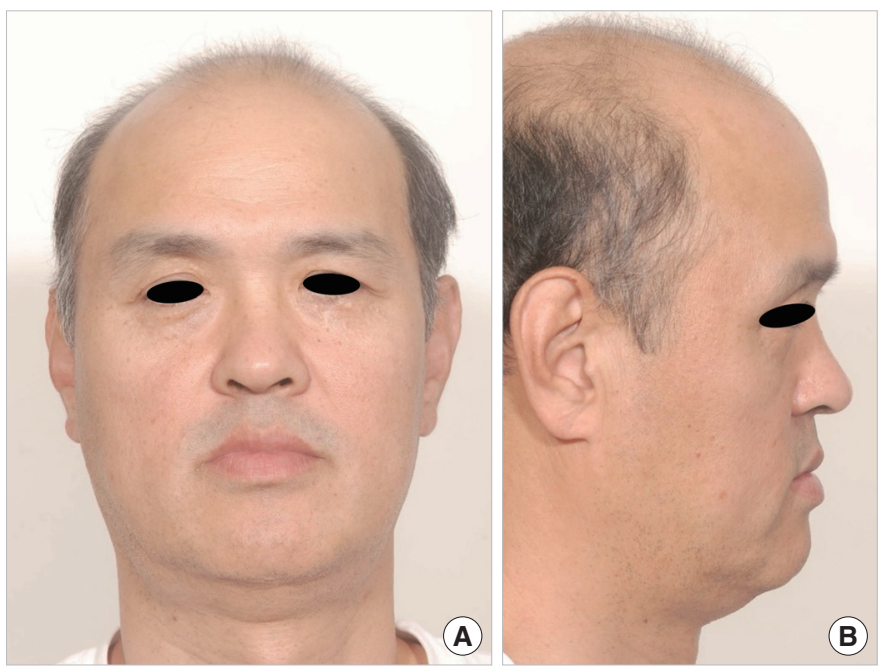

Fig. 1. Preoperative gross appearance of the patient. Note the midface depression, concave facial profile, and anteroposterior discrepancy of the upper and lower lips. (A) Frontal view and (B) lateral view.
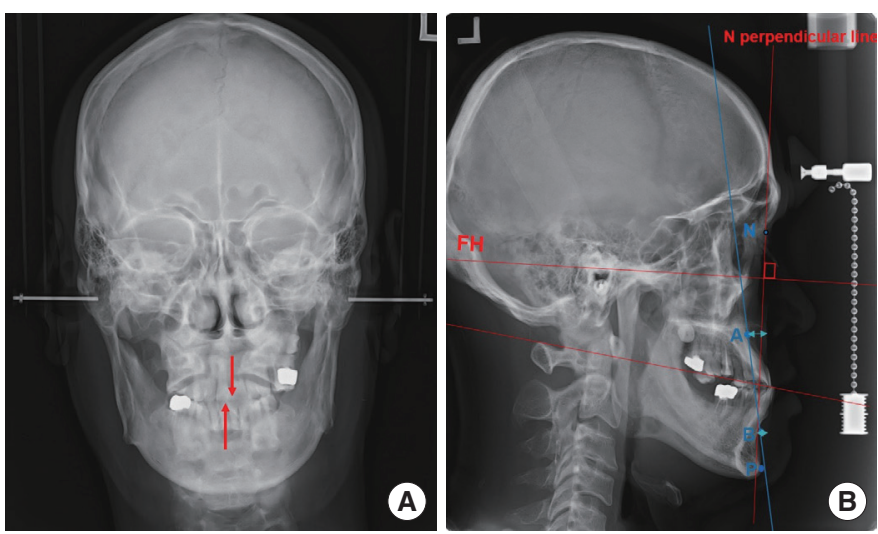

Fig. 2. Preoperative cephalometric analysis. FH to AB angle was larger than normal value. (A, B) Note the skeletal class III malocclusion, inadequate position of maxilla with depression, midline shifting (red arrows), and canting. A, A point; B, B point; FH, Frankfort horizontal; N, nasion. status, and senile appearance due to depression of the maxilla and less visible incisor, and maxillary advancement with down through Le Fort I osteotomy was planned (Tables 1 and 2, Fig. 4). We excluded other surgical options such as Le Fort III osteotomy or twojaw surgery by considering the above-mentioned factors. The surgical treatment objective was planned considering the elongation of the left side, impaction of the right side, and the correction of canting and midline shifting. Surgery was performed through in-

Table 1. Preoperative cephalometric analysis

\begin{tabular}{lccc}
\hline Preoperative & Right & Center & Left \\
\hline Vertical $(\mathrm{mm})$ & & & \\
Central incisor & - & 94.08 & - \\
1st molar & 92.04 & - & 93.01 \\
Horizontal (mm) & & & \\
Central incisor & 67.87 & 67.89 & 67.91 \\
Transverse (mm) & & & \\
Central incisor & - & 38.04 & - \\
1st molar & 69.58 & - & 6.91 \\
\hline
\end{tabular}

Table 2. Postoperative cephalometric analysis

\begin{tabular}{lccc}
\hline Postoperative & Right & Center & Left \\
\hline Vertical (mm) & & & \\
Central incisor & & $95.98(\downarrow 1.90)$ & \\
1st molar & $91.90(\uparrow 0.14)$ & & $93.89(\downarrow 0.88)$ \\
Horizontal (mm) & & & \\
Central incisor & 71.42 & $71.23(3.34)$ & 71.03 \\
Transverse (mm) & & & \\
Central incisor & & $40.70(\leftarrow 2.66)$ & \\
1st molar & $73.34(\leftarrow 3.76)$ & & $10.87(\leftarrow 3.96)$ \\
\hline
\end{tabular}

Le Fort I osteotomy with total advancement and midline correction to right. $3.34 \mathrm{~mm}$ advancement, $2.66 \mathrm{~mm}$ midline correction to right, $1.90 \mathrm{~mm}$ elongation based on upper left central incisor, $0.14 \mathrm{~mm}$ impaction based on upper right second molar, 0.88 elongation based on upper left first molar.
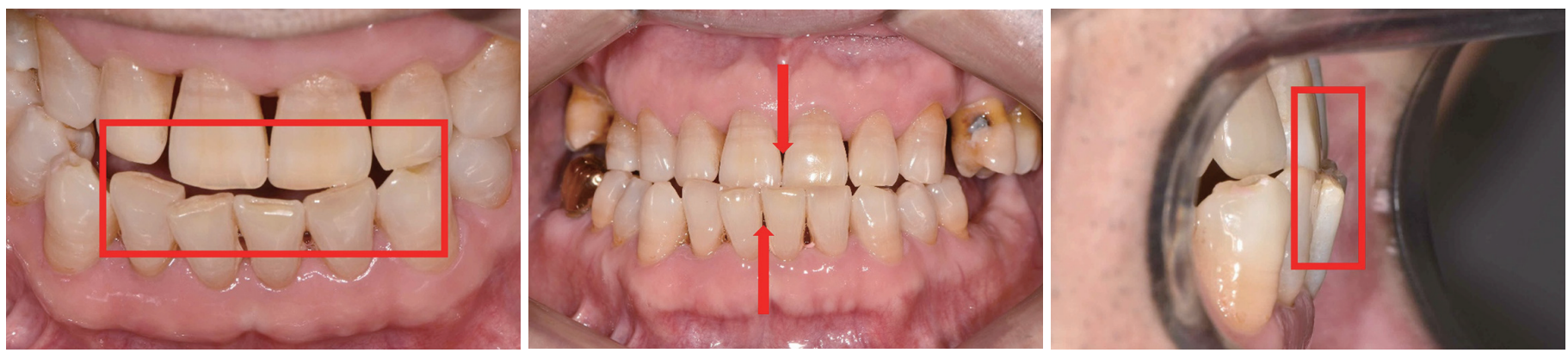

Fig. 3. Preoperative intraoral photography. Note the class III malocclusion, crossbite (squares), and midline shift (arrows) to left of the maxillary plane. 

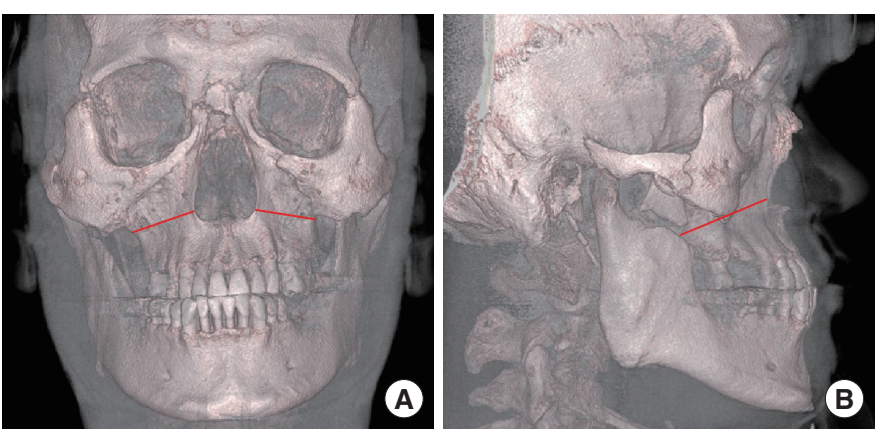

Fig. 4. Preoperative three-dimensional computed tomography. Le Fort I osteotomy Note displacement of the maxilla due to inadequate reduction and fixation at the time of injury. (A) Frontal view and (B) lateral view; the red line indicating the line that performed the Le Fort I osteotomy.

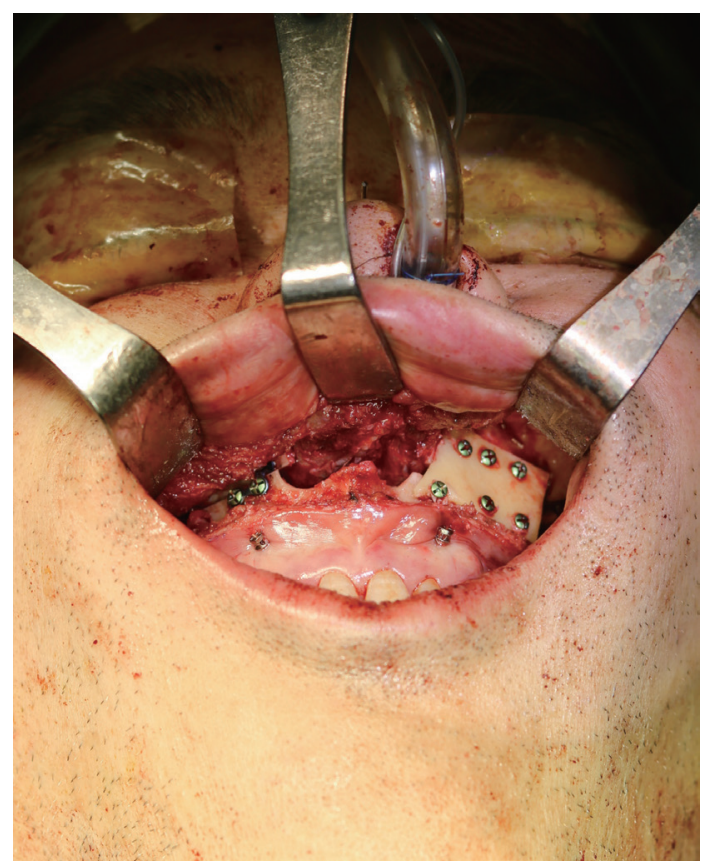

Fig. 5. The cortical bone was harvested from the right mandible angle, inset to the bony gap, and fixed with miniscrews.

traoral incision; a fracture line that was due to previous trauma was observed, and some drilling marks suspected as signs of improper reduction and internal fixation were also observed. After full mobilization of the maxilla through Le Fort I osteotomy, the lower segment of the maxilla was advanced to fit with preoperative wafers, and fixation was performed using a 2.0-mm titanium miniplates and miniscrews. After Le Fort I osteotomy, the bony gap on the left side was measured at approximately $3.0 \mathrm{~mm}$. The author decided to perform bone graft. The cortical bone was harvested from the right mandible angle, inset to the bony gap, and fixed with miniscrews (Fig. 5). Alar flaring due to advancement of the maxilla was corrected using an alar cinching suture. Postoperative inter-
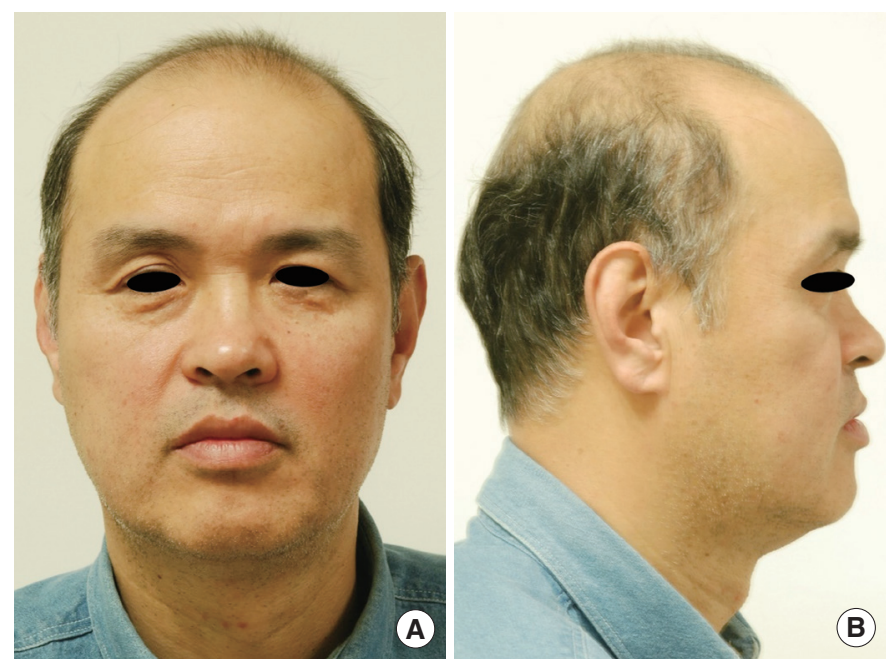

Fig. 6. Postoperative gross appearance of the patient at 3 months after surgery. Note the more convex contour of the midface and youthful appearance. (A) Frontal view and (B) lateral view.
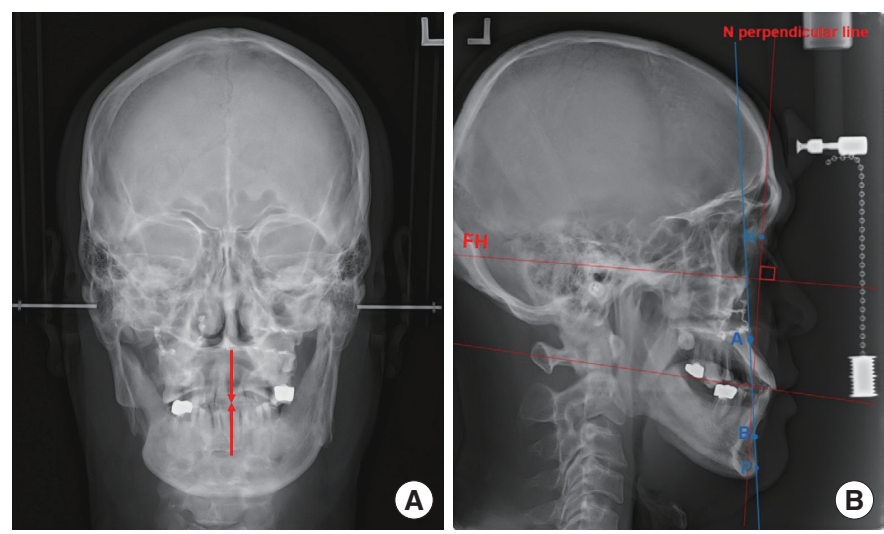

Fig. 7. Postoperative cephalometric analysis showed that the reference angles were closer to the ideal angles. (A, B) Note the correction of the midline shifting (red arrows). FH, Frankfort horizontal plane; N, nasion; A, A point; B, B point.

maxillary fixation was maintained for 4 weeks, and orthodontic treatment was not performed. Postoperative results were evaluated by gross appearance, cephalometric analysis, and postoperative intraoral imaging. The gross appearance of the patient did not have a dramatic effect but had more convex contour before surgery. As a result, senile-looking facial appearance became more youthful (Fig. 6). Postoperative cephalometric analysis showed that the reference angles representing relative mutual positions of the maxilla and mandible were closer to the ideal angles. Canting of the maxillary plane and midline shifting were also corrected (Fig. 7). Three months postoperatively, the patient returned to normal occlusion, and the patient reported subjective improvement of occlusion (Fig. 8). 

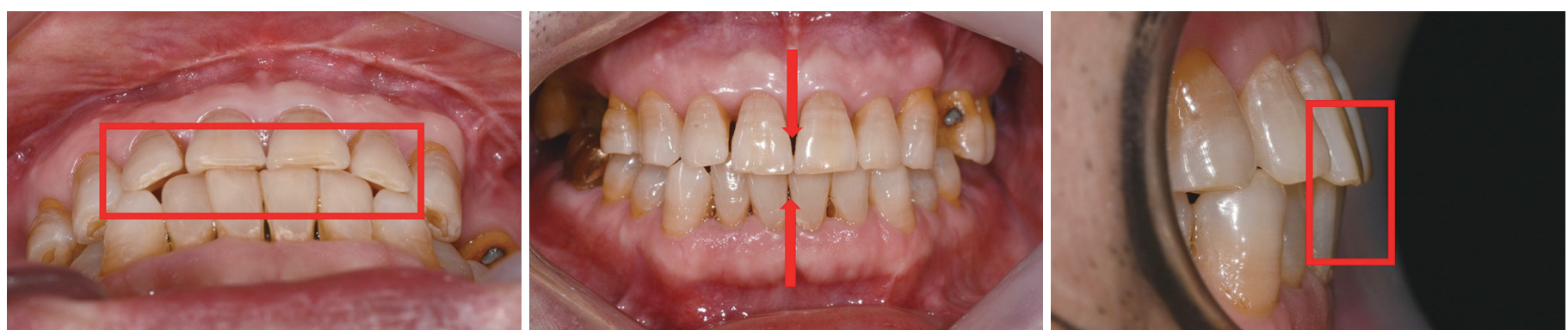

Fig. 8. Three months postoperatively, normal occlusion was restored (red squares). Also, note the correction of the midline shifting and canting of the maxilla (red arrows).

\section{DISCUSSION}

Trauma in the modern society is characterized by multiple injuries, and fatal damage is often accompanied by facial bone fracture. However, because facial bone fracture is not a life-threatening problem, surgery for facial bone fractures may be delayed if other life-threatening morbidities associated with trauma are present and should be resolved first. Although open reduction is performed at an appropriate time, if the fracture fragments are comminuted, reduction of the fracture to its anatomical position before trauma is difficult, and firmly fixing it is difficult. Delayed surgery and inadequate reduction often result in malunion of the fracture and other functional problems [1-3]. Le Fort fracture not only deforms the facial aesthetic harmony, but also causes functional abnormalities, such as malocclusion and speech problems. The purpose of treatment for patients with malocclusion due to Le Fort fracture is to maintain and restore both function and aesthetics. The author experienced the case of malocclusion caused by delayed, improper treatment of Le Fort III fracture. The patient both had Le Fort III and II fractures and had not only craniofacial dysjunction, but also midface deformity. In maxillary fracture, the maxillary arch is posteriorly displaced, and the mandible is relatively protruded and consequently has class III malocclusion. A relatively friable maxilla also has a junction with a relatively strong skull base. Most maxillary fractures move the maxilla backward, while the maxillary molar moves the mandible downward, causing anterior open bite [1]. Because Le Fort fracture causes various problems in occlusion, focusing on the restoration of both appearance and function to find a way to maintain its function are necessary. The traditional surgical correction of class III malocclusion and mandibular prognathism is set back and repositioning of the mandible [4,5]. If the set back of the mandible is performed, the position of the mandible may be appropriate in cephalometric analysis. However, because the volume of the mandible is reduced, and the position is set back, the outline of the lower face becomes less clear. To maintain a good corrected occlusal position without causing deformity in the temporomandibular joint, the maxillary and mandibular teeth should be in harmony with the centric relationship while maintaining maximum occlusal contact. If class III malocclusion is corrected through mandibular set back, obtaining the centric relationship and maximal occlusal contact of the occlusion becomes more difficult. Correction of the depression of the mid-face and concavity of the lateral appearance with mandibular set back are also impossible. Le Fort I osteotomy can improve the appearance of the midface. Teuscher and Sailer [6] reported that the upper lip and nasal tip could be advanced at an average of $6.76 \mathrm{~mm}$ and $1.46 \mathrm{~mm}$, respectively, with anterior relocation in the case of Le Fort I osteotomy.

Fundamentally, the patient's appearance and occlusion were the result of a Le Fort III fracture; hence, Le Fort III osteotomy was required to regain the pre-traumatic facial appearance as closely as possible. In another approach, two-jaw surgery was required in conjunction with orthodontic treatment to improve both malocclusion and facial appearance. The author did not perform Le Fort III osteotomy because of following reasons: first, the patient was bald. Second, the patient did not want to undergo surgery with high morbidity. Third, because the patient also had a Le Fort II fracture, the zygomatic arch could migrate and could cause another facial asymmetry if midline shift correction was attempted through Le Fort III osteotomy. The authors' initial plan included two-jaw surgery with orthodontic treatment to improve both occlusion and facial appearance. Two-jaw surgery was not considered because of the following: first, the patient did not want orthodontic treatment. Second, it was a constant dentition without dental compensation for many years after the onset of post-traumatic malocclusion. The patient's oral and facial bone condition was not appropriate for Le Fort III osteotomy and two-jaw surgery. Therefore, the author considered the patient's facial appearance, occlusion status, and senile appearance due to depression of the maxilla and less visible incisor, and maxillary advancement with down through Le Fort I osteotomy was planned. The surgical treatment objective was planned considering the elongation of the left side, impaction of the right side, and the correction of canting and midline shifting. In cephalometric analysis, this patient had lower face protrusion that was anthropometrically judged to be well anterior or anteriorly divergent to the ideal position. Thus, the author performed maxillary advancement as a paradoxical correction of class III malocclu- 
sion. The advantages of using maxillary advancement alone compared with two-jaw surgery are (1) Le Fort I osteotomy is a simpler procedure; (2) the recurrence rate is lower; or (3) the recovery of root injuries is rapid, and infraalveolar nerve damage and morbidity, such as temporomandibular joint dysfunction can be minimized [7]. In addition, Le Fort I osteotomy alone can induce clockwise rotation, counterclockwise rotation, and advancement, elongation, correction of midline shifting, and canting by differentiating the direction of osteotomy and the amount of ostectomy. Rosen [8] reported that mandibular setbacks reduce lower facial skeletal volume and may result in inappropriate soft tissue support, resulting in soft tissue excess and loss of skeletal definition. For mild-tomoderate mandibular prognathism, maxillary advancement alone provides sufficient aesthetic improvement, and functional correction is possible $[7,8]$. This approach may also have some limitations. Not all class III malocclusions can be corrected with maxillary advancement. Rosen [8] showed that mandibular set back is an indication of moderate mandibular prognathism less than 6.9 $\mathrm{mm}$ in discrepancy. In severe cases, mandibular set back should also be performed to improve the appearance and to enhance bone stability. Also, if a Le Fort III fracture is corrected by Le Fort I osteotomy alone, depression of the nasofrontal region can be more pronounced. Treatment of malocclusion should improve the occlusal comfort and aesthetic aspects of the patient while maintaining the stability of the corrected occlusion and optimal oral health. The authors conclude that Le Fort I osteotomy correction could be a good option among variable correction methods of traumatic class III malocclusion, and the results were satisfactory in functional and cosmetic aspects.

\section{CONFLICT OF INTEREST}

No potential conflict of interest relevant to this article was reported.

\section{ETHICAL APPROVAL}

The study was performed in accordance with the principles of the Declaration of Helsinki.

\section{PATIENT CONSENT}

The patient provided written informed consent for the publication and the use of his images.

\section{ORCID}

Han Byeol Jin (https://orcid.org/0000-0002-0965-4372) Jee Hyeok Chung (https://orcid.org/0000-0003-2782-8898)

Kyung Sik Kim (https://orcid.org/0000-0002-2101-9654)

Seung Hong Kim (https://orcid.org/0000-0001-8092-8551)

Joon Choe (https://orcid.org/0000-0002-0860-3231)

Jeong Yeol Yang (https://orcid.org/0000-0002-4343-4429)

\section{REFERENCES}

1. Kim KT, Jung SH, Yun SH, et al. The surgical correction of post-traumatic malocclusion. J Korean Soc Plast Reconstr Surg 1998;25:613-21.

2. Furnas DW. Transverse maxillary osteotomy for malunion of maxillary fractures. Plast Reconstr Surg 1968;42:378-83.

3. Lancaster LL Jr, Quinn JH, Fournet LF, et al. Treatment of malunited fractures of the mandible and maxilla. J Oral Surg 1970;28:310-5.

4. Hunsuck EE. A modified intraoral sagittal splitting technic for correction of mandibular prognathism. J Oral Surg 1968;26:250-3.

5. Hebert JM, Kent JN, Hinds EC. Correction of prognathism by an intraoral vertical subcondylar osteotomy. J Oral Surg 1970;28:651-3.

6. Teuscher U, Sailer HF. Stability of Le Fort I osteotomy in class III cases with retropositioned maxillae. J Maxillofac Surg 1982;10:80-3.

7. Han KW, Ye CH, Kang JS. Maxillary advancement for mandibular prognathism. J Korean Soc Plast Reconstr Surg 1996;23:690-701.

8. Rosen HM. Maxillary advancement for mandibular prognathism: indications and rationale. Plast Reconstr Surg 1991;87:823-32. 